\title{
Electrical Storm in a Patient with Dilated Cardiomyopathy Suppressed with Cardiac Resynchronization Therapy
}

\author{
Dimitris Tsiachris, MD¹, Odysseas Kaitozis, MD², Skevos Sideris, MD², Polychronis Dilaveris, MD', \\ Konstantinos Gatzoulis, MD ${ }^{1}$ \\ ${ }^{1}$ University of Athens, First Cardiology Clinic, Athens, Greece \\ ${ }^{2}$ Hippokration Hospital, Cardiology Department, Athens, Greece
}

\begin{abstract}
We present the case of a 55-year-old man with dilated cardiomyopathy and a syncopal episode who developed electrical storm with multiple instances of both monomorphic and polymorphic sustained ventricular tachycardia terminated by shocks in the face of worsening heart failure not responding to triple antiarrhythmic drug therapy including sedation and intubation in the coronary care unit. Hemodynamic improvement after cardiac resynchronization therapy with defibrillation backup (CRT-D) implantation brought about a dramatic response in the situation. This case report demonstrates the usefulness and efficiency of CRT-D as an option of treating electrical storm by improving ventricular function in the case of electrical instability being an expression of severe pump failure.

Copyright @ 2015 Science International Corp.
\end{abstract}

\section{Key Words}

Cardiac resynchronization therapy • Electrical storm • Ventricular tachycardia

\section{Introduction}

A worrying incidence of proarrhythmic effect in the form of electrical storm (ES) after the institution of cardiac resynchronization therapy (CRT) has been repeatedly reported in the recent past [1]. However there are contradictory reports related to either the

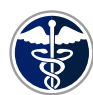

Fax +1 2037853346

E-Mail: agr@scienceinternational.org

http://arrhythmiagr.com/ (c) 2015 Arrhythmia Grand Rounds

Published by Science International Corp. ISSN 2326-4012

Accessible online at: http://arrhythmiagr.com/ proarrhythmic [1] or the antiarrhythmic $[2,3]$ potential of the CRT as far as the induction or/and suppression of ES. In line with these reports, we present a case of ES in a patient with dilated cardiomyopathy not responding to triple antiarrhythmic drug therapy, including sedation and intubation, but successfully suppressed after biventricular pacing.

\section{Case Report}

A 55-year-old man with a history of psoriasis and dilated cardiomyopathy was referred to our clinic for the treatment of palpitations and a syncopal episode. His functional status was worsening New York Heart Association (NYHA) class III on optimal medical treatment with ACE-inhibitors, b-blockers, diuretics, and aldosterone antagonists. Amiodarone was also prescribed for a 2-month history of paroxysmal atrial fibrillation and discontinued 1 month prior to his admission for the syncopal episode. The resting electrocardiogram (ECG) showed a normal sinus rhythm with a prolonged PR interval of $210 \mathrm{~ms}$, a wide QRS duration of $184 \mathrm{~ms}$ with a left bundle branch block (LBBB) pattern and left axis deviation, as well as a prolonged QT interval of $490 \mathrm{~ms}$ (QT corrected $=550$ ms) (Figure 1). A 24-hour Holter monitoring exhibited more than 3000 monomorphic premature ventricular

* Corresponding Author:

Konstantinos A Gatzoulis, MD

First Cardiology Clinic

University of Athens

Mail box 175, Drafi Attikis 19009, Athens, Greece

Tel.: (0030)6944580369, fax: (0030)2132088676, E-Mail: kgatzoul@med.uoa.gr 
contractions and a mean heart rate of $80 \mathrm{bpm}$ without episodes of bradycardia, heart block or polymorphic ventricular tachycardia (VT).

The two-dimensional echocardiogram documented a marked left ventricular (LV) dysfunction (LV ejection fraction, LVEF of $25 \%$ ) with a diffuse hypokinesia and increased dimensions of the LV, whereas the right ventricle presented normal dimensions and global function. The results of urine, laboratory tests, including electrolytes, and arterial blood gases were within normal ranges.

An invasive electrophysiological study (EPS) under metoprolol use revealed a normal sinus node function with significant conduction system disease, consisting of atrial-His ( $\mathrm{AH}$ ) interval at $114 \mathrm{~ms}$ and a His-ventricular (HV) interval prolonged at $94 \mathrm{~ms}$. During programmed ventricular stimulation of the right ventricular outflow tract, a sustained polymorphic VT causing syncope was induced with triple ventricular extrastimuli and was terminated by shock.

The following days the patient went into ES with multiple and increasingly frequent episodes of both sustained monomorphic VT as well as polymorphic VT degenerating into ventricular fibrillation (VF) interrupted by repeated shocks. Amiodarone, xylo- caine, metoprolol, and deep sedation with intubation were required in order to better control the ES. However the VT/VF episodes continued on the next 4 days. Based on telemetry analysis there were no episodes of bradycardia-related VT since heart rate prior to every VT episode exceeded $60 \mathrm{bpm}$ in all cases.

At this stage we hypothesized that the multiple arrhythmic episodes were the result of the deterioration of the unstable electrophysiologic substrate as an expression of severe cardiac pump failure and that improving ventricular function by CRT would probably reduce ventricular arrhythmia burden [4].

A CRT with defibrillation capacity (CRT-D) was thus implanted. The procedure was uneventful without any complications. Sensing and pacing thresholds were optimal. Two days after the implantation an additional episode of VT was interrupted with the first attempt of antitachycardia pacing. Pre-implantation antiarrhythmic drug administration caused a further prolongation of QRS (195 ms) and corrected QT intervals (560 ms).

Eighteen months later the patient gained improvement of activities in daily life and is now in NYHA class II. The LVEF increased from $25 \%$ to $30 \%$ but most importantly he is free of ventricular arrhythmic events as

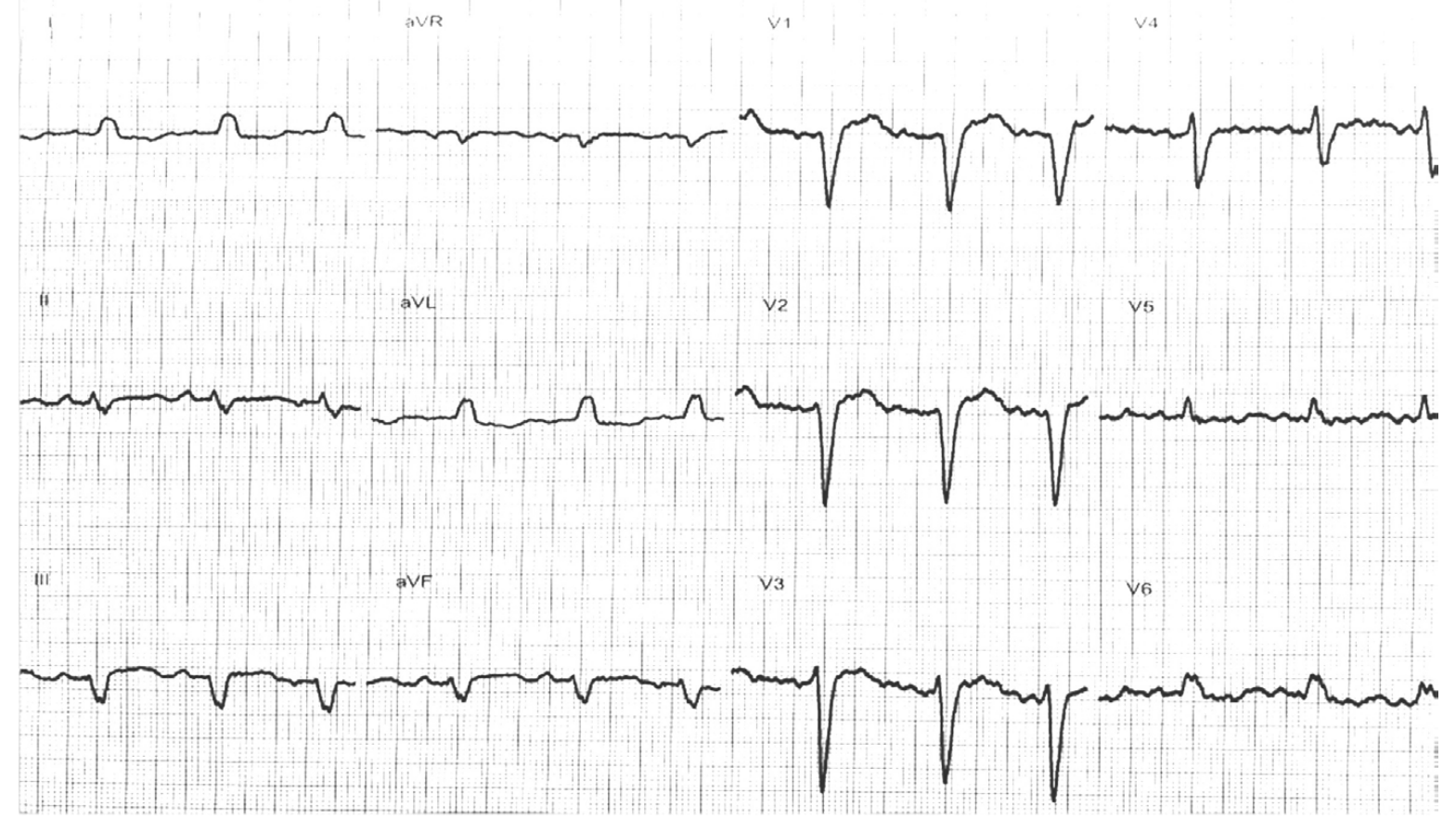

Figure 1. Initial (prior to amiodarone administration) 12 lead ECG showing LBBB with left axis deviation and borderline first-degree atrioventricular block. 
no VT/VF episodes were recorded. Post-implantation QRS was $180 \mathrm{~ms}$ and corrected QT interval was 520 ms. Treatment with amiodarone remained for the first 12 months and discontinued thereafter (Figure 2).

\section{Discussion}

CRT has been well established to have a favorable impact on all-cause mortality and re-hospitalization for heart failure. The current guidelines suggest CRT-D therapy in order to reduce morbidity and mortality in patients with dilated cardiomyopathy with NYHA function class III or IV despite optimal pharmacological treatment, LVEF less than or equal to $35 \%$, sinus rhythm (SR), LBBB, and QRS duration greater than or equal to $120 \mathrm{~ms}$.

Nevertheless the effect of biventricular pacing on arrhythmia events has not been as clear cut. There are a number of reports suggesting that CRT can cause arrhythmia or even induction of electrical storm after the implantation of biventricular pacing [1]. This has been attributed to the altered ventricular activation sequence and the prolongation of ventricular repolarization phase induced by the LV pacing [1]. This is of particular concern in cases when CRT device implantation is not associated with a defibrillator backup. The incidence reported in limited single series is low, between $3.4 \%$ and $4 \%$, with predominance in ischemic cardiomyopathy. On the other hand there is strong evidence that CRT may improve several arrhythmogenic indices in both ischemic and dilated cardiomyopathy patients [5]. In line with these studies, MADIT-CRT trial documented a lower incidence of malignant ventricular tachyarrhythmias in patients responding to biventricular pacing. It has been suggested that reverse LV mechanical remodeling is associated with reversal of electrical remodeling and a lower rate of appropriate defibrillator therapy following CRT.

Similar to our case there are also two other case reports of ES suppression by biventricular pacing in patients with dilated cardiomyopathy and VT [2, 3]. It has also been suggested that the incidence of ES is lower among patients treated with a CRT-D as compared to those treated with an implantable cardiac defibrillator [4]. The full effect of biventricular pacing on the arrhythmogenic substrate of heart failure and especially its acute effect on ES is yet to be fully understood. Focusing on our case, we may recognize

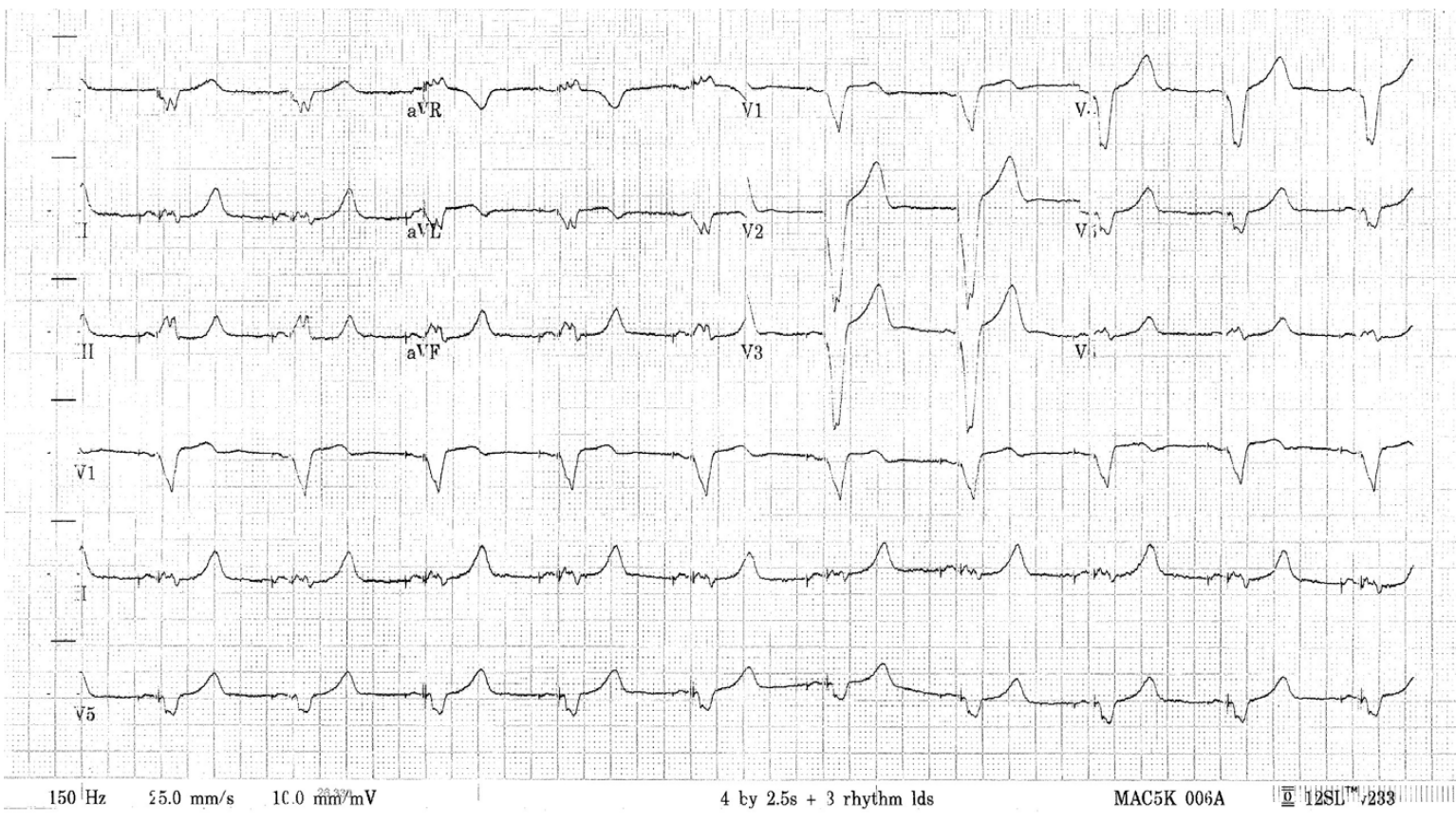

Figure 2. Twelve months postimplant 12 lead ECG showing LBBB with right axis deviation and atrial pacing attributed to sick sinus syndrome secondary to amiodarone. 
three different mechanisms that in parallel acted as ES suppressants. Beyond the hemodynamic improvement and the mild QT reduction related with biventricular pacing, late effect of amiodarone (which was administered systematically for the 12 months) might also contribute towards this direction. Our observations suggest that CRT can be an option of treating ES by improving ventricular function in the case of electrical instability being an expression of severe pump failure.

\section{References}

1. Vouliotis $A H$, Tsiachris $D$, Dilaveris $P$, Sideris S, Gatzoulis K. Cardiac resynchronization therapy and proarrhythmia: Weathering the storm. Hospital Chronicles. 2012;7:234-240. DOI: 10.1111/j.15408167.2008.01151.x

2. Tanabe $Y$, Chinushi M, Washizuka T, Minagawa $S$, Furushima $H$, Watanabe $H$, et al. Suppression of electrical storm by biventricular pacing in a patient with idiopathic dilated cardiomyopathy and ventricular tachycardia. Pacing Clin Electrophysiol. 2003;26:101-102. DOI: 10.1046/j.1460-9592.2003.00156.x

3. Nicoletti I, Tomei R, Zanotto G, Dalla Vecchia E, Zorzi E, Vassanelli C. The beneficial effect of biventricular pacing on ventricular tachycardia in a patient with non ischemic cardiomyopathy. Int J Cardiol. 2008;126:2931. DOI: 10.1016/j.ijcard.2007.01.013

4. Gatzoulis KA, Andrikopoulos GK, Apostolopoulos T, Sotiropoulos E, Zervopoulos G, Antoniou J, et al. Electrical storm is an independent predictor of adverse long term outcome in the era of implantable defibrillator therapy. Europace. 2005;7:184-192. DOI: 10.1016/j.eupc.2005.01.003

5. Dilaveris P, Giannopoulos G, Synetos A, Aggeli C, Raftopoulos L, Arsenos P, et al. Effect of biventricular pacing on ventricular repolarization and functional indices in patients with heart failure: lack of association with arrhythmic events. Europace. 2009;11:741750. DOI: 10.1093/europace/eup094
Cite this article as: Tsiachris D, Kaitozis O, Sideris S, Dilaveris P, Gatzoulis K. Electrical Storm in a Patient with Dilated Cardiomyopathy Suppressed with Cardiac Resynchronization Therapy. Arrhythmia Grand Rounds 2015; 1 (Issue 1): 7-10. DOI: http://dx.doi.org/10.12945/j. agr.2015.00011-14 